\title{
The Interpretation of Disjunction in the Scope of Dou in Child Mandarin
}

\author{
Shasha $\mathrm{An}^{1 *}$, Peng Zhou ${ }^{2}$ and Stephen Crain ${ }^{3}$ \\ ${ }^{1}$ Center for Linguistics and Applied Linguistics, Faculty of English Language and Culture, Guangdong University of Foreign \\ Studies, Guangzhou, China, ${ }^{2}$ Department of Foreign Languages and Literatures, Tsinghua University, Beijing, China, \\ ${ }^{3}$ Department of Linguistics, Macquarie University, Sydney, NSW, Australia
}

\section{OPEN ACCESS}

Edited by:

Dieter Hillert,

San Diego State University,

United States

Reviewed by:

Weiyi Ma,

University of Arkansas, United States Katsuo Tamaoka,

Nagoya University, Japan

*Correspondence:

Shasha An

anshasha2015@sina.com

201840004@oamail.gdufs.edu.cn

Specialty section:

This article was submitted to

Language Sciences,

a section of the journal

Frontiers in Psychology

Received: 23 September 2020

Accepted: 24 November 2020

Published: 15 December 2020

Citation:

An S, Zhou P and Crain S (2020)

The Interpretation of Disjunction

in the Scope of Dou in Child

Mandarin. Front. Psychol. 11:609492.

doi: 10.3389/fpsyg.2020.609492
A recent theory provides a unified cross-linguistic analysis of the interpretations that are assigned to expressions for disjunction, Negative Polarity Items, Free Choice Items, and the non-interrogative uses of wh-phrases in languages such as Mandarin Chinese. If this approach is on the right track, children should be expected to demonstrate similar patterns in the acquisition of these linguistic expressions. Previous research has found that, by age four, children have acquired the knowledge that both the existential indefinite renhe "any" and wh-words in Mandarin Chinese are interpreted as Negative Polarity Items when they are bound by downward entailing operators, but the same expressions are interpreted as Free Choice Items (with a conjunctive interpretation) when they are bound by deontic modals (Mandarin keyi) or by the Mandarin adverbial quantifier dou "all". The present study extends this line of research to the Mandarin disjunction word huozhe. A Truth Value Judgment Task was used to investigate the possibility that disjunction phrases that are bound by the adverbial quantifier dou generate a conjunctive interpretation in the grammars of Mandarin-speaking 4-year-old children. The findings confirmed this prediction. We discuss the implications of the findings for linguistic theory and for language learnability.

Keywords: child Mandarin, disjunction, wh-words, dou, free choice items, negative polarity items

\section{INTRODUCTION}

Recently a theory has been advanced that provides a unified semantic analysis of disjunction, Negative Polarity Items (NPIs), Free Choice Items (FCIs), and the non-interrogative interpretations of wh-phrases across languages (Fox, 2007; Chierchia, 2013). The present study tests the unification account directly, by asking if Mandarin disjunctive phrases evoke conjunctive inferences when they are bound by the quantificational adverb (hereafter, Q-adverb) dou. This possibility is investigated in an experimental study with Mandarin-speaking children and adults. The main finding confirms the prediction, and provides circumstantial support for the unified account.

We begin with a brief tour of the semantics of existential expressions, so-called $\exists$-items. Three English $\exists$-items, the existential indefinite some, the disjunction word or, and the polarity sensitive expression any, are illustrated in examples (1) - (4).

(1) Someone coughed.

(2) Ted or Gen coughed.

(3) Sally didn't hear anyone cough.

(4) If Sally hears someone/Ted or Gen/anyone cough, she breaks out the cold medicine. 
Sentence (1), someone coughed, is true if and only if there exists an individual $\mathrm{x}$ such that $\mathrm{x}$ coughed. In a domain with just two individuals, Ted and Gen, (1) is logically equivalent to the disjunctive statement in (2), Ted or Gen coughed (e.g., Crain and Khlentzos, 2010). The polarity sensitive expression any is another $\exists$-item (e.g., Klima, 1964; Baker, 1970; Ladusaw, 1979, 1980; Carlson, 1980; Linebarger, 1987; Horn, 1989; Krifka, 1995; among others). Sentence (3) Sally didn't hear anyone cough is true only if there does not exist an individual $x$ such that Sally heard $\mathrm{x}$ cough. In the domain with two individuals, Ted and Gen, this statement is true only if Sally didn't hear Ted cough and didn't hear Gen cough ${ }^{1}$. Example (4) illustrates the logical equivalence under discussion. In a domain with just two individuals, all three of the $\exists$-items we have discussed are licensed in the antecedent of a conditional statement.

In addition to their use as existential expressions ( $\exists$-items), the English disjunction word or and the polarity sensitive expression any also generate free choice/conjunctive inferences in certain linguistic contexts (for discussion of or, see Kamp, 1973; Zimmermann, 2000; Geurts, 2005; Fox, 2007; Barker, 2010; Chierchia, 2013; for discussion of any, see Quine, 1960; Vendler, 1967; Horn, 1972; Lasnik, 1972; Ladusaw, 1979; Carlson, 1981; Dayal, 1998). Both the disjunction word or and the polarity sensitive expression any license free choice inferences when they appear in the scope of a deontic modal, such as English may (Mandarin keyi) (Lasnik, 1972; Kamp, 1973; Lee and Horn, 1994; Dayal, 1995; Zimmermann, 2000; Geurts, 2005; Barker, 2010; Zhou et al., 2013; Huang and Crain, 2014, 2020). This is illustrated in (5) and (6).

(5) Kung Fu Panda may drive the green car or the orange car.

(6) Kung Fu Panda may drive any of the cars.

Sentences in (5) and (6) both convey the message that Kung Fu Panda (hereafter, KFP) is free to choose among the available cars. According to the unified account, the disjunction word or and the polarity expression any are initially analyzed as $\exists-$ items in sentences as (5) and (6). The fact that these sentences generate free choice/conjunctive inferences is due to a process called recursive exhaustification, which we discuss next.

\section{Recursive Exhaustification}

The theoretical proposal by Fox (2007), Chierchia (2013) attempts to provide a unified analysis of $\exists$-items, all of which give rise to free choice/conjunctive inferences via recursive exhaustification. In the following we demonstrate how the free choice reading of disjunction is derived via recursive exhaustification.

(7) KFP may drive the green car or the orange car.

\footnotetext{
${ }^{1}$ The relationship between disjunction and existential quantification was made explicit in Kalish and Montague (1964). In logic texts, the symbol for disjunction is "V." Kalish and Montague (1964) adopted an enlarged disjunction symbol "V" to represent the existential quantifier (the symbol " $\exists$ " in other logic texts). Similarly, to capture the relationship between conjunction and universal quantification, Kalish and Montague represented the universal quantifier using an enlarged version of the conjunction symbol " $\wedge$ ", namely " $\Lambda$ " (rather than " $\forall$ ").
}

The sentence in (7) can be paraphrased as a conjunctive statement: KFP may push the green car and KFP may push the orange car (for discussion, see Kamp, 1973; Geurts, 2005; Fox, 2007; Barker, 2010; Chierchia, 2013; Zhou et al., 2013; Zimmermann, 2000). We will render the meaning of "may" symbolically using the possibility operator, $\diamond$. The generalization that disjunctive statements yield conjunctive truth conditions is represented by the inference pattern in (8).

$$
\text { (8) } \diamond(\mathrm{p} \vee \mathrm{q}) \sim>\diamond \mathrm{p} \wedge \diamond \mathrm{q}
$$

The fact that the inference in (8) is legitimate is surprising, because a plain disjunctive sentence, i.e., one without a modal, never conveys the corresponding conjunctive inference. In fact, it typically conveys its negation. That is, from the statement KFP pushed the green car or the orange car, it does not follow that KFP pushed both cars. An explanation for the inference in (8) has been advanced by Fox (2007) and by Chierchia (2013). We will follow Chierchia's formulation of the account, which involves a recursive application of an algorithm akin to that of a scalar implicature. The algorithm is referred to as recursive exhaustification because an exhaustivity (ONLY) operator is applied to its own output.

As in a typical scalar implicature, the algorithm compares the statement made by a speaker with alternative statements that the speaker might have made. According to the algorithm in question, however, these alternative statements are enriched by the exhaustivity operator, to include their associated (negative) inferences. These are inferences that would have been attributed to the speaker if these alternative statements had been produced, instead of the actual statement. At the first step in the algorithm, then, the exhaustivity operator generates inferences that enrich the alternatives to what the speaker said. Then, the exhaustivity operator (ONLY) applies a second time, in order to eliminate those enriched alternatives that are stronger than what the speaker actually said. Having sketched the general idea, we will now provide a brief overview of the two steps involved in recursive exhaustification, using the disjunctive statement in (7), repeated here as (9).

(9) KFP may drive the green car or the orange car. $\diamond(p \vee q)$

At the first step, the assertion is compared to its "subdomain" alternatives. These subdomain alternatives are formed using these disjuncts in the predicate phrase of the original assertion, as shown in (10) and (11). These subdomain alternatives are compared to the assertion at the second step in the algorithm, but only after they have been enriched with their associated inferences.

(10) KFP may drive the green car. subdomain alternative $=\diamond p$.

(11) KFP may drive the orange car. subdomain alternative $=\diamond \mathrm{q}$.

What are the inferences associated with (10) and (11)? When a speaker asserts (9), the question under discussion is which cars KFP has been given permission to drive. The green car and the orange car are the relevant alternatives. Suppose that the speaker had asserted one of the subdomain alternatives, (10) or (11), instead of (9). If the speaker had asserted (10), this would 
have invited the inference in (12). Similarly, from the subdomain alternative in (11), we would have inferred (13).

(12) KFP may drive the green car, but not the orange car. $\diamond \mathrm{p} \wedge$ $\neg \diamond \mathrm{q}$

(13) KFP may drive the orange car, but not the green car. $\diamond \mathrm{q} \wedge$ $\neg \diamond \mathrm{p}$

The intuition of such inferences is as follows. Suppose your friend Mary asks who coughed in a situation with only two individuals, Ted and Gen. Another friend, Bruce, answers - Ted. We interpret Bruce's answer as shorthand for Only Ted coughed. That is, we infer from Bruce's fragment answer, Ted, that Gen did not cough. To account for this kind of inference, we posit that an implicit exhaustivity operator, ONLY, is operative in such discourse sequences that include disjunctive statements. This exhaustivity operator is responsible for generating the pragmatic inferences that enrich the subdomain alternatives (10) and (11), as illustrated in (14) and (15). This is the first step in the process of recursive exhaustification. It is schematically represented in (16).

(14) ONLY [KFP may drive the green car] $\sim>$ KFP may drive the green car, but not the orange car. $\diamond \mathrm{p} \wedge \neg \diamond \mathrm{q}$

(15) ONLY [KFP may drive the orange car]

$\sim>$ KFP may drive the orange car, but not the green car. $\diamond \mathrm{q} \wedge \neg \diamond \mathrm{p}$

(16) 1st Exhaustification:

a. ONLY $[\diamond \mathrm{p}]=\diamond \mathrm{p} \wedge \neg \diamond \mathrm{q}$ [ $\diamond \mathrm{p}$ is a subdomain alternative, with inference $\neg \diamond \mathrm{q}]$

b. ONLY $[\diamond \mathrm{q}]=\diamond \mathrm{q} \wedge \neg \diamond \mathrm{p}$

$[\diamond \mathrm{q}$ is a subdomain alternative, with inference $\neg \diamond \mathrm{p}]$

At the second step in the algorithm, the enriched alternatives are stacked up against the original disjunctive sentence KFP may push the green car or the orange car, which is cast symbolically as $\diamond(\mathrm{p} \vee \mathrm{q})$. We refer to the enriched alternatives as the "scalar alternatives" to the original assertion. The second step in the algorithm determines whether or not each of the scalar alternatives is stronger than the original statement made by the speaker. In the example under consideration, the scalar alternatives to $\diamond(\mathrm{p} \vee \mathrm{q})$ are $\diamond \mathrm{p} \wedge \neg \diamond \mathrm{q}$ and $\diamond \mathrm{q} \wedge \neg \diamond \mathrm{p}$. If a scalar alternative is stronger than the speaker's statement, then we make the usual inference associated with scalar implicatures; that is, we infer that the speaker was not in a position to assert the scalar alternative, so we infer the negation of the scalar alternative. It can easily be verified that both the scalar alternatives under consideration are stronger than the original disjunctive statement. The scalar alternative $\diamond \mathrm{p} \wedge \neg \diamond \mathrm{q}$ is stronger than the original assertion $\diamond(\mathrm{p} \vee \mathrm{q})$, and so is $\diamond \mathrm{q} \wedge$ $\neg \diamond$ p. Consequently, we infer their negations: $\neg[\diamond \mathrm{p} \wedge \neg \diamond \mathrm{q}]$ and $\neg[\diamond \mathrm{q} \wedge \neg \diamond \mathrm{p}]$. This is the second step in the recursive exhaustification algorithm process. This step is represented in words in (17), and the remaining steps of the derivation are indicated symbolically in (18).

(17) ONLY [KFP may drive the green car or the orange car]. a. $\sim>$ It is false that KFP may drive the green car but may not drive the orange car, and

b. $\sim>$ It is false that KFP may drive the orange car but may not drive the green car.

(18) 2nd Exhaustification
a. $\quad \mathrm{ONLY}[\diamond(\mathrm{p} \vee \mathrm{q})]=\diamond[\mathrm{p} \vee \mathrm{q}]$
b. $\neg[\diamond \mathrm{p} \wedge \neg \diamond \mathrm{q}]$
c. $\neg[\diamond \mathrm{q} \wedge \neg \diamond \mathrm{p}]$
(negation of scalar alternative)
c.
d. $\diamond \mathrm{p} \Leftrightarrow \diamond \mathrm{q}$
(negation of scalar alternative) (c), and the definition of $\Leftrightarrow)$ (from (b) and
e. $\diamond[\mathrm{p} \vee \mathrm{q}] \wedge \diamond \mathrm{p} \Leftrightarrow \diamond \mathrm{q}=\diamond \mathrm{p} \wedge \diamond \mathrm{q} \quad$ (from (a) and $(\mathrm{d}))$

From (17-a), it follows that if KFP may drive the green car, then he may also drive the orange car (18-b), and from (17-b), it follows that if KFP may drive the orange car, then he may also drive the green car $(18-c)$. Therefore, if he is permitted to drive either one of the cars, he is also permitted to drive the other one. Together with the original statement - KFP may drive the green car or the orange car - the fact that KFP may drive either car if and only if he may drive the other. (18d) entails that KFP may drive the green car and he may drive the orange car (18e). This completes our overview of the recursive exhaustification algorithm.

\section{Dou in Mandarin \& Predictions for Child Language}

The Q-adverb dou (roughly English "all”) in Mandarin Chinese is known for its multi-functions (for reviews, see Zhou and Crain, 2011; Xiang, 2020). One of the well-known functions of dou is an FCI licenser, which converts $\exists$-items, for example, preverbal renhe "any"-expressions and the non-interrogative use of wh-words into free choice inferences (Xiang, 2016, 2020). In view of the semantic parallelism between different $\exists$-items, it is pertinent to understand how the Q-adverb dou converts wh-phrases/renhe "any" into conjunctive interpretations, before we make predictions about the interpretation of disjunction associated with the Q-adverb dou. We use sentences with whwords and ones with the FCI renhe in Mandarin as examples. When the wh-phrase shenme che precedes dou, as in (20), the whexpression generates a conjunctive/free choice inference, just as the FCI renhe "any" does in (19). Both (19) and (20) have the same meaning - KFP is free to choose which of the cars to drive. If the Q-adverb dou is removed, however, the FCI renhe is no longer tolerated, resulting in the ungrammatical sentence (21). Moreover, in the absence of the Q-adverb dou, wh-phrases no longer generate free choice inferences, so example (22) can only be interpreted as a $w h$-question. 
(19) Renhe che KFP dou keyi kai any car KFP DOU may drive

Literal meaning: "Any car KFP DOU may drive."

Intended: "KFP may drive any car."

(20) Shenme che KFP dou keyi kai any car KFP DOU may drive

Literal meaning: "What car KFP DOU may drive."

Intended: "KFP may drive any car."

(21) *Renhe che KFP keyi kai any car KFP may drive

"KFP may drive any car."

(22) *Shenme che KFP keyi kai what car KFP may drive

"What car may KFP drive?"

The fact that $w h$-words and the FCI renhe are assigned a conjunctive interpretation is attributed to recursive exhaustification. A brief outline of how the recursive exhaustification algorithm derives a universal reading for the $w h$-word in sentences with dou is sketched in (23). We will use sentence (20) as our example.

(23) a. $\mathrm{WH} \exists \mathrm{x} \in\{\mathrm{a}, \mathrm{b}, \mathrm{c}\}$, car KFP DOU may drive.

b. $[a \vee b \vee c]$ car KFP DOU may drive.

c. [DOU $[\mathrm{a} \vee \mathrm{b} \vee \mathrm{c}]]$ car KFP may drive.

d. $[\mathrm{a} \wedge \mathrm{b} \wedge \mathrm{c}] \mathrm{car}$ KFP may drive.

e. $\mathrm{wH}_{\mathrm{x}} \forall \mathrm{x} \in\{\mathrm{a}, \mathrm{b}, \mathrm{c}\}$ car KFP may drive.

In the process, the Q-adverb dou plays a role, which is in analogy to the exhaustivity (ONLY) operator in recursive exhaustification algorithm (Xiang, 2016). The characteristic of the Q-adverb dou makes Mandarin Chinese a natural and sound laboratory to test the unified analysis of $\exists$-items proposed by Fox (2007) and Chierchia (2013).

The derivation of free choice inferences of $w h$-words in association with dou is as follows. First, wh-words, such as shenme che, are analyzed as existential indefinites. In (23), the existential expression ranges over the available cars in the domain of discourse, so we can render the meaning of the whword shenme che as $+\mathrm{WH} \exists \mathrm{x} \in\{\mathrm{a}, \mathrm{b}, \mathrm{c}\}$. The meaning of the wh-word is then converted into a disjunction, [a $\vee \mathrm{b} \vee \mathrm{c}$ ], which is logically equivalent. Then, the recursive exhaustification algorithm applies, as described in the previous section. However, in this algorithm, the overt quantifier dou replaces the covert exhaustivity operator (only) and applies to the disjunction phrase $[a \vee b \vee c]$. The output of the algorithm is a conjunction [a $\wedge \mathrm{b}$ $\wedge c]$, which is the source of the universal force attributed to the sentence, $\forall \mathrm{x} \in\{\mathrm{a}, \mathrm{b}, \mathrm{c}\}$.

We have seen that, in a finite domain, disjunctive phrases are the logical equivalents of the free choice item renhe, which logically equals to the $\exists$-items $w h$-words. We will see (in the literature review) that the free choice item $w h$-words receive a conjunctive/universal interpretation in sentences with dou. Therefore, it is a straightforward prediction that disjunction phrases in Mandarin are expected to generate a conjunctive inference when they are bound by the adverbial quantifier dou. This prediction was investigated in the present study. As far as we know, this prediction has not been previously verified, in either adult or child Mandarin.

To test the prediction, the disjunctive phrase must appear to the left of the Q-adverb dou, and there cannot be any intervening plural noun phrases $^{2}$. These are prerequisites to the study, because dou is typically associated with a plural NP to its left. These conditions are satisfied in sentences like (24), where the nearest NP to the left of dou is the singular name, gongfuxiongmao "KFP."

(24) Jiaozi huozhe shousi, gongfuxiongmao dou hui zuo. dumplings or sushi KFP DOU can make Literal meaning: "Dumplings or sushi, KFP DOU can make."

Intended: "KFP can make dumplings and sushi."

If the recursive exhaustification algorithm applies to (24), as expected, the following conjunctive interpretation should be a paraphrase of the meaning of (24): KFP can make dumplings and sushi. Mandarin-speaking children's interpretation of sentences such as (24) will be investigated in our experiment, which we will turn to momentarily. However, before we report on the details of the experiment, it will be useful to review the findings of previous research on the acquisition of $\exists$-items by preschoolaged children.

\section{LITERATURE REVIEW}

This section reviews previous experimental studies of the acquisition of existential expressions, focusing mainly on Mandarin Chinese. We will briefly sketch the findings of the studies that investigated children's interpretation of three kinds of sentences: Mandarin sentences with the disjunction operator huozhe and the deontic modal verb keyi "may"; English sentences with a deontic modal verb and the FCI any, and its Mandarin counterpart renhe; and we will discuss Mandarin sentences with wh-words and the Q-adverb dou, such that we will add Mandarin $w h$-words to our stockpile of $\exists$-items. These studies demonstrate that 4-year-old Mandarin-speaking children draw free choice/conjunctive inferences from disjunctive statements that contain the deontic modal operator keyi "may" (Zhou et al., 2013), and derive free choice inferences from the noninterrogative uses of $w h$-words associated with the Q-adverb dou "all" (Zhou and Crain, 2011; Zhou, 2015). Moreover, 4-year-old native English and Mandarin children have acquired the dual interpretations of "any" and its Mandarin counterpart renhe, respectively (Tieu, 2010; Huang and Crain, 2014).

\section{Free Choice Inferences of Disjunction in Child Language}

Zhou et al. (2013) demonstrated that 4-year-old Mandarinspeaking children $(4 ; 1-4 ; 9, M=4 ; 3)$ draw free choice inferences

\footnotetext{
${ }^{2}$ As noted by Xiang $(2016,2020)$, to license the universal free choice use of disjunction, dou must be followed by a possibility modal, for example, one of the Mandarin counterparts of "can." Since the major concern of this study is with the linguistic elements preceding dou, we will not go into details about Xiang's theories. Interested readers are referred to the references for more details.
} 
for disjunction phrases that occur in the scope of the deontic modal keyi "may." In the study, children were asked to judge sentences like (25) in a context in which KFP was only given permission to drive the green car, but not the orange car.

$$
\begin{array}{cccc}
\text { Gongfuxiongmao } & \text { keyi } & \text { kai } & \text { lvse } \\
\text { KFP } & \text { may } & \text { drive } & \text { green } \\
\text { xiaoche } & \text { huozhe } & \text { jvse } & \text { xiaoche. } \\
\text { car } & \text { or } & \text { orange } & \text { car }
\end{array}
$$

Literal meaning: "KFP may drive the green car or the orange car."

Intended: "KFP may drive the green car and the orange car."

If children compute a free choice/conjunctive inference for the disjunctive phrase in sentence (25), then they should judge it to be a false description of the story, because the sentence means that KFP was granted permission to drive both the green car and the orange car. Children's judgments were exactly as predicted. Based on this finding, the authors concluded that children generate free choice inferences for disjunctive phrases that appear in the scope of the deontic modal keyi "may."

\section{Free Choice Inferences of Any in Child Language}

Tieu (2010) investigated the interpretations of any assigned by English-speaking preschool children $(0 ; 11,04-5 ; 02,12)$, both in linguistic contexts that license NPIs and in linguistic contexts that license FCIs. To assess this, Tieu surveyed the transcripts of the spontaneous speech of 40 monolingual English-speaking children using the CHILDES database (MacWhinney, 2000). Twenty-six of the 40 children produced 15 or more instances of any in linguistic contexts that license NPIs with few errors. These children's productions of any emerged at the same time in declarative and interrogative contexts, whereas any emerged significantly later in linguistic contexts that license FCIs. That is, preschool English-speaking children have the knowledge of both NPI any and FCI any.

Mandarin renhe behaves much like its English counterpart any; it can appear both in linguistic contexts that license NPIs, and in contexts that license FCIs. Huang and Crain (2014) investigated the interpretation assigned to renhe by 4-6 years old Mandarin-speaking children (4;5-6;3, $M=5 ; 4)$. Sentences as in (26), in which renhe appeared in sentences with the modal expression neng "can", and sentences as in (27) where renhe was omitted were tested. The finding was that the child participants assigned a conjunctive interpretation to sentences that contained renhe, such as (26), but not to sentences without renhe, as in (27). That is, children judged (26) to mean that KFP can push any one of the three cars that were made available in the experimental workspace, whereas children judged (27) to mean that KFP can only push a single car.

$$
\begin{aligned}
& \begin{array}{c}
\text { Gongfuxiongmao } \\
\text { KFP }
\end{array} \text { can tuidong renhe } \\
& \text { yi-ge } \\
& \text { one-CL chezi. }
\end{aligned}
$$

(27)

Gongfuxiongmao neng tuidong yi-ge chezi.
KFP

"KFP can push one of the cars."

a. KFP can push any car.

b. KFP can push the green car or the orange car or the purple car.

c. KFP can push the green car, and can push the orange car, and can push the purple car.

In combination with neng "can", renhe "any" invokes a universal reading. The universal reading can be derived from an existential, such as Mandarin renhe "any", by the same recursive exhausitification algorithm we described earlier. The beginning and end points of the derivation are as follows. The experimental workspace had three cars (green, orange and purple). Symbolically we can represent "neng... renhe" statements (e.g., 26) as $\diamond \exists x \in\{\mathrm{p}, \mathrm{q}, \mathrm{r}\}$. This is logically equivalent to the disjunctive statement $\diamond(\mathrm{p} \vee \mathrm{q} \vee \mathrm{r})$, which serves as the input to the recursive exhaustification algorithm. The output of the algorithm is a conjunctive statement, $\diamond \mathrm{p}$ $\wedge \diamond \mathrm{q} \wedge \diamond \mathrm{r}$, which is logically equivalent to the universal, $\diamond \forall \mathrm{x} \in\{\mathrm{p}, \mathrm{q}, \mathrm{r}\}$. In other words, the process can be represented as in (28).

\section{Wh-Words and the Q-Adverb Dou in Child Mandarin}

The next series of experimental studies we review investigated children's interpretation of Mandarin wh-words in combination with the Q-adverb dou.

The first experiment we reviewed is carried out by Zhou (2015), which assessed Mandarin-speaking children's (3;6-4;9, $M=4 ; 3$ ) knowledge of the existential interpretation of $w h$-words in contexts that license NPIs, as in (29), and in contexts such as (30), where only the interrogative use of $w h$-words is licensed.

(29) Meiyou xiongmao chi shenme
not-have phuiguo.
"No panda ate any fruit."
(30)
Mei-zhi xiongmao dou chi-le
every-CL phenme
shuiguo?
fruit
"What kind of fruit did every panda eat?"

In (29), the predicate phrase of the negative (downward entailing) quantificational expression meiyou xiongmao "no panda" licenses the existential reading of the wh-word shenme. By contrast, the positive (upward entailing) predicate phrase of the quantificational expression meizhi xiongmao "every panda" in (30) does not license the existential reading of a wh-word. On a typical trial, three pandas were eating breakfast. All of them took one strawberry, but none of them picked a lemon. Both children and adults rejected (29) 100\% of the time in this context, and they justified their responses by pointing out that every panda ate some fruit. In response to the sentence in (30), both children and adults interpreted 
the sentence as a question, and responded with the answer "strawberry."

A second experiment in the same study investigated children's understanding of the universal/conjunctive interpretation of $w h$ words in Mandarin with test sentences as in (31) and (32). For adults, the quantificational adverb dou is required to license the universal reading of $w h$-phrases. This is illustrated in (31) and (32). In (31), the wh-word shei "who" is bound by dou, thereby yielding a universal reading. By contrast, the sentence in (32) is a wh-question, because the wh-word shei is not bound by dou.

Shei dou tiao-guo-le fangzi.
Who DOU jump-over-ASP house
"Everyone jumped over the house."
Shei tiao-guo-le fangzi?
who jump-over-ASP house
"Who jumped over the house?"

In a typical story there were three horses: a white horse, a black horse and a yellow horse. The horses engaged in a jumping competition. The white horse and the black horse easily jumped over a house, but the yellow horse was not as successful. Following the story, a puppet produced one of the test sentences; half of the children heard (31), and half heard (32). Both children and adults rejected sentenced like (31) $95 \%$ of the time, and they justified their rejections by pointing out the yellow horse didn't jump over the house. In response to sentence (32), both children and adults consistently provided the answer to a question, usually answering "the white horse and the black horse." The author interpreted the findings as evidence that preschool Mandarin-speaking children have the knowledge of the non-interrogative uses of whwords.

Another study by Zhou and Crain (2011) investigated children's sensitivity to the structural position of the Q-adverb dou and a wh-word using so-called dou-conditionals. If a whword appears in the antecedent of a dou-conditional, and is followed by dou (in the consequent clause), the wh-word generates a conjunctive interpretation ("whoever"). On the other hand, wh-words that are preceded by dou function as whquestion markers. This contrast is illustrated in (33) and (34). Although both examples contain the wh-word shei "who" and the quantificational adverb dou, due to these licensing conditions, the example in (33) is a statement, whereas the sentence in (34) is a question. Mandarin-speaking children (3;5-5;0, $M=4 ; 3)$ were tested their interpretation of sentences like those in (33) and (34). In the story corresponding to (33) and (34), three villagers (a pig, a rabbit, and a dog) were being harassed by a menacing crocodile. The village head, Mr. Owl, called upon his superhero friends, Spiderman and Batman, to chase away the crocodile. After the superheroes had chased away the crocodile for the third and last time, a puppet presented (33) to one group of children, and (34) to a different group of children.

$\begin{array}{cccc}\text { Eyu } & \text { qu } & \text { yao } & \text { shei, } \\ \text { crocodile } & \text { go } & \text { bite } & \text { who, } \\ \text { maotouying } & \text { cunzhang } & \text { dou } & \text { zhao-le } \\ \text { owl } & \text { village-head } & \text { DOU } & \text { find-ASP }\end{array}$

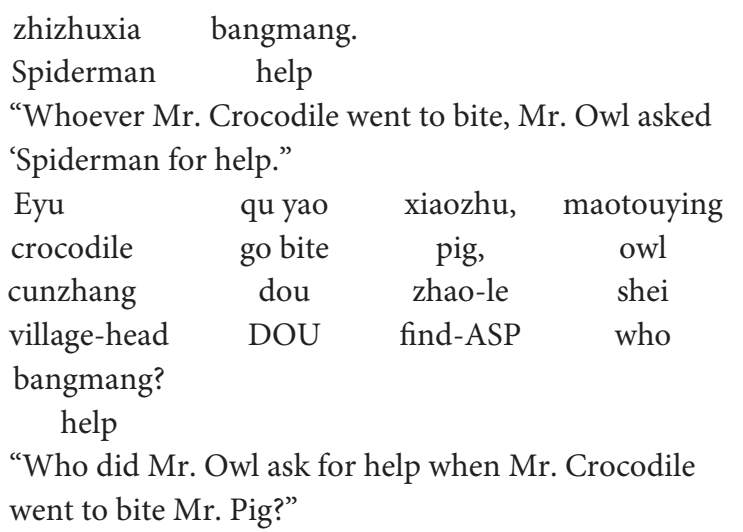

The finding was that 37 of the 42 children consistently interpreted sentences like (33) as statements, and all 42 children interpreted sentences like (34) as questions, so they responded with the appropriate answer (e.g., "Batman"). This finding provides compelling evidence that young Mandarin-speaking children know the licensing conditions on $w h$-words appearing with the quantificational adverb dou.

In this section, we first reviewed previous research on children's acquisition of the existential expressions any in English and renhe "any" in Mandarin Chinese. Then we reviewed the findings of studies in which children were asked to interpret $w h$ words in sentences with the Q-adverb dou, versus ones without dou or ones in which dou preceded the wh-word. The findings demonstrated that both young English-speaking and Mandarinspeaking children have acquired the dual interpretations of a variety of existential expressions at a very early age. In the present study, we extend this line of research to the Mandarin disjunction word huozhe. We were interested to see whether young Mandarin-speaking children interpret the disjunctive phrases as having a conjunctive reading when they are bound by the adverbial quantifier dou.

\section{EXPERIMENT 1}

This experiment was devised to assess whether or not the presence of dou in the sentence converts disjunctive phrases into conjunctive meanings in child Mandarin. A typical minimal pair of test sentences is illustrated in (35) and (36).

(35)

$\begin{array}{cccc}\text { jiaozi } & \text { huozhe } & \text { shousi, } & \text { gongfuxiongmao } \\ \text { dumplings } & \text { or } & \text { sushi } & \text { KFP } \\ \text { dou } & \text { hui } & \text { zuo. } & \\ \text { DOU } & \text { can } & \text { make } & \end{array}$

Literal meaning: "Dumplings or sushi, KFP DOU can make."

Intended: "KFP can make dumplings and sushi."

$\begin{array}{cccc}\text { jiaozi } & \text { huozhe } & \text { shousi, } & \text { xiaoxiongmaomen } \\ \text { dumplings } & \text { or } & \text { sushi } & \text { little pandas } \\ \text { dou } & \text { hui } & \text { zuo. } & \\ \text { DOU } & \text { can } & \text { make } & \end{array}$

Literal meaning: "Dumplings or sushi, little pandas DOU can make.” 
Intended: "The little pandas all can make dumplings, or sushi."

The unified account predicts that, when a disjunctive phrase occurs in the scope of the adverbial quantifier dou, the disjunctive phrase is converted into a conjunctive interpretation, via recursive exhausitification. This applies to sentences such as (35), where the Q-adverb dou takes scope over the disjunctive phrase jiaozi huozhe shousi "dumplings or sushi." In this sentence, dou cannot take scope over the (singular) proper noun KFP, so the disjunctive phrase is the only option. Therefore, the sentence in (35) is expected to mean that KFP can make dumplings AND sushi. In (36), by contrast, there is a plural noun to dou's left, xiaoxiongmaomen "little pandas." Therefore, it is anticipated that the sentence means all of the pandas can make dumplings OR sushi. For brevity, we will refer to sentences like (35) as Type 1, and to sentences like (36) as Type 2.

Our experimental hypothesis is: in Type 1 sentences like (35), in which the disjunctive phrase occurs in the scope of the adverbial quantifier dou, children will give the disjunctive phrase a conjunctive interpretation; in Type 2 sentences like (36), in which dou takes scope over the plural NP, children will interpret the disjunctive phrase with disjunctive truth conditions.

\section{Participants}

We tested 30 Mandarin-speaking children (4.18-4.90, $M=4.65)$ on their interpretation of Type 1 and Type 2 sentences. In addition, 30 Mandarin-speaking adults $(18-22, M=19.32)$ were tested as controls. All of the participants have not been reported as having developmental or psychiatric disorders, language or hearing impairments.

\section{Procedures}

All of the adult participants and caregivers of child participants were informed of the contents and purposes of the study prior to the experiment. Adult and child participants (or their caregivers) provided written consent and oral consent before the practice trials. The procedures were in accordance with the ethical guidelines with the Declaration of Helsinki (World Medical Association General Assembly, 1964) and its later amendments or comparable ethical standards.

\section{Methods}

The methodology we adopted was a Compute-based version of the Truth Value Judgment Task (TVJT). Originally, the Truth Value Judgment Task (TVJT) is designed to investigate the range of interpretations children assign to sentences (Crain and Thornton, 1998). The task involves two experimenters. One experimenter acts out short stories in front of child participants, using toys and props. The second experimenter plays the role of a puppet, who watches the stories alongside the child. At the end of each story, the puppet explains what he thinks happened in the story, using one of the test sentences. The child's task is to judge whether or not the puppet said the right thing about the story. Child participants were assured that the puppet sometimes makes mistakes when he tries to describe what happened in the story. If the child judges the puppet's statement to be incorrect, then the experimenter asks the child to explain to the puppet "what really happened" in the story. This allows the experimenters to verify that the child is rejecting the test sentences correctly. In this study, we prerecorded stories and test trials, and presented them to participants on a laptop. This helps to keep the test materials consistent between the child participants.

The child participants were introduced to the task and tested individually in a quiet room in their school. Before the test part was introduced, two practice trials were administered to familiarize the children with the task. On one practice trial, the puppet uttered a sentence that was true in the context and; on the other practice trial, the puppet said something false. This reinforced the idea that the puppet didn't always pay attention and would sometimes say something incorrect about what had happened in the stories. Only those children who gave correct judgments to the two practice trials were included in the experiment. Adult controls were tested with a written form of the test materials.

\section{Materials}

Eight stories were created. After each story, there were two test sentences and two filler sentences. One typical trial is used to illustrate the test scenarios of the present study.

KFP and his five little panda friends attended a cooking school. After one week of classes, they had completed the course. They came to KFP's place to show their cooking skills.

Kung Fu master was curious to know what they could cook with only one week of lessons. He asked: "KFP, what can you make now?"

KFP: "Why don't you guess? First, let me give you a hint. Dumplings and sushi. I can cook one of them."

Master: "It is definitely dumplings, because your favorite food is dumplings."

KFP nodded his agreement.

Kung Fu master went on and asked the little pandas: "Did you guys learn how to make dumplings and sushi?"

They replied: "No. It's a shame. We did not have enough time to learn how to make both of them. All of us have learnt how to make one kind of them. We all learnt the same one. Do you know which one we can make?"

The sentences in (37)-(40) were produced after the story. Example (37) means that KFP can make dumplings AND sushi. But in the corresponding context KFP can only make dumplings, participants were, therefore, expected to reject (37) by making reference to the fact that KFP cannot make sushi. By contrast, participants should accept (39), since it is a correct description of what happened in the story. To remind the participants of the events that had taken place in the story, the scene presented in Figure 1 was visible to the child participants.

\begin{tabular}{|c|c|c|c|}
\hline \\
\hline \multicolumn{4}{|l|}{$\begin{array}{l}\text { larget Sentence } \\
\text { jiaozi }\end{array}$} \\
\hline dumplings & or & sushi & \\
\hline gongfuxiongmao & dou & hui & zuo. \\
\hline KFP & DOU & can & make \\
\hline \multicolumn{4}{|c|}{$\begin{array}{l}\text { Literal meaning: "Dumplings or sushi, KFP DOU } \\
\text { can make." }\end{array}$} \\
\hline \multicolumn{4}{|c|}{ Intended: "KFP can make dumplings and sushi." } \\
\hline
\end{tabular}




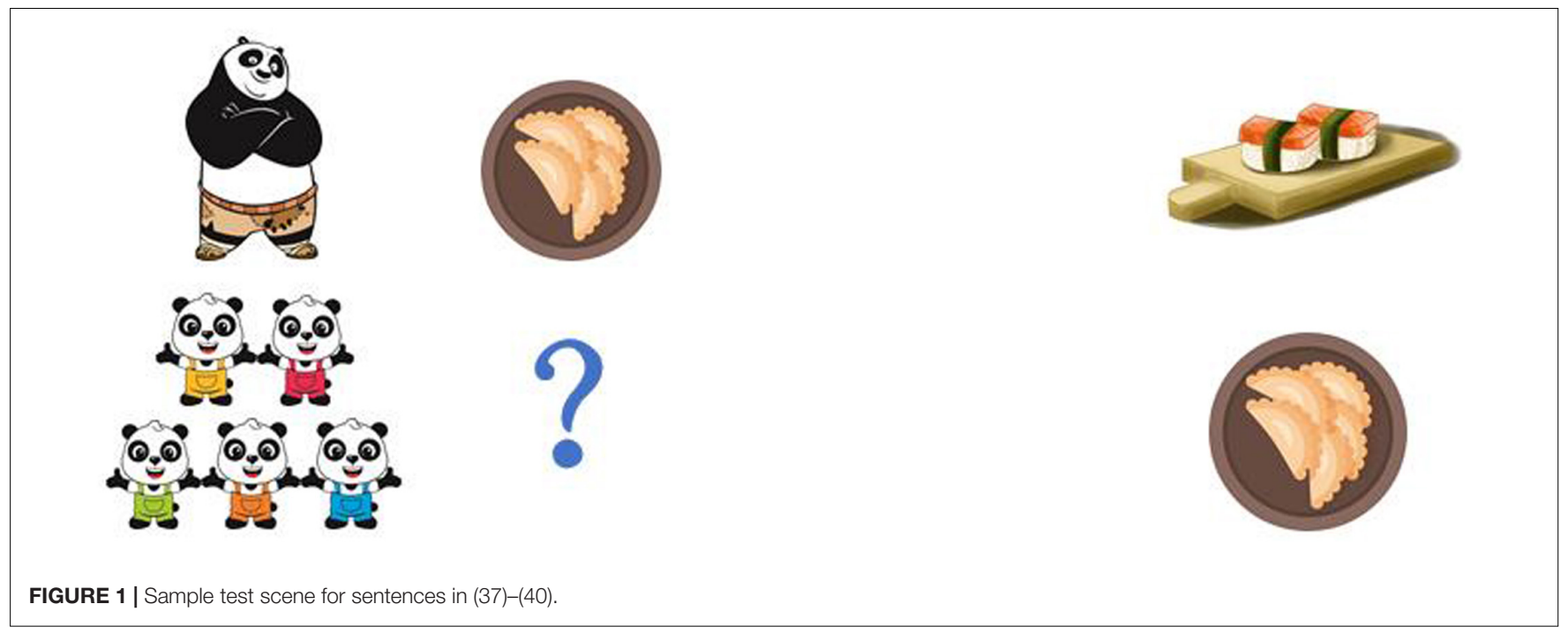

(38)

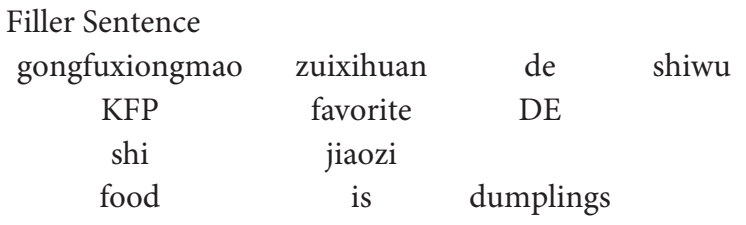

"KFP's favorite food is dumplings."

(39)

Target Sentence

$\begin{array}{cccc}\text { jiaozi } & \text { huozhe } & \text { shousi, } & \\ \text { dumplings } & \text { or } & \text { sushi } & \\ \text { xiaoxiongmaomen } & \text { dou } & \text { hui } & \text { zuo. } \\ \text { little pandas } & \text { DOU } & \text { can } & \text { make } \\ \text { Literal meaning: "Dumplings or sushi, little pandas }\end{array}$

DOU can make."

Intended: "The little pandas all can make dumplings, or sushi."

(40)

$\begin{array}{ccc}\begin{array}{c}\text { Filler Sentence } \\ \text { gongfuxiongmao }\end{array} & \text { you } & \text { liu-ge } \\ \text { KFP } & \text { have } & \text { six-CL } \\ \text { hao } & \text { pengyou. } & \\ \text { good } & \text { friends } & \end{array}$

"KFP has six good friends."

\section{Results}

The software package of SPSS 26 was utilized to analyze the data. Percentages of correct judgments from each participant were calculated for the test trials and filler trials. All of the participants accepted the four true filler sentences, and rejected the four false filler sentences. Therefore, we did not carry out further analysis with the data of filler trials. Turning to the test sentences, children rejected Type 1 test sentences $87.5 \%$ of the time and the control group of adults rejected them at a rate of $91.7 \%$. On the trial showcased above, for example, the children justified their rejections of (37) by explicitly referring to the fact that gongfuxiongmao buhui zuo shousi ("KFP cannot make sushi") or by pointing at the sushi, meaning that KFP could not make sushi. Data analysis revealed no significant differences between children and adults in the proportion of correct responses, $p=0.37$. In response to Type 2 test sentences, such as (39), child participants and adults accepted them $90.8 \%$ of the time and $90.4 \%$ of the time, respectively. No significant difference has been found in the proportion of the correct responses by children and adults, $p=0.93$.

The experimental findings suggest that like adults, 4year-old Mandarin-speaking children have the knowledge that dou quantifies over disjunctive phrases to its left, thereby giving disjunction phrases a conjunctive interpretation in such sentences.

\section{EXPERIMENT 2}

This experiment, which serves as a follow-up test, was included to assess the reliability of the results of Experiment 1. Specifically, we were interested to find out whether adults' interpretation of the test sentences would vary without the corresponding contexts in Experiment 1.

\section{Participants}

Four groups (Group 1, Group 2, Group 3, Group 4) of Mandarin-speaking adults were tested. All of them were college students, whose age ranged from 18 to 22 and had not been reported as having developmental disorders, language or learning difficulties. Each group consisted of 63 participants. We compared the mean age of each group and found no significant difference between groups, $p=0.12$.

\section{Procedures}

All of the participants were informed of the purposes of the study and provided written consent to take part in the experiment. The procedures were in accordance with the ethical guidelines with 
the Declaration of Helsinki (World Medical Association General Assembly, 1964) and its later amendments or comparable ethical standards.

\section{Methods}

Participants were presented audio-recorded sentences. After that, they were asked to judge the truth value of following utterances according to the firstly presented sentences. If they believe the utterances were not correct, they were required to provide their justification.

\section{Materials}

In this experiment, each trial contained three utterances. After listening to the first utterances, participants were required to indicate whether the following utterances they heard were correct or not by putting down their answers on an answer sheet. To avoid potential carryover effect, the second and third utterances following the target sentences were presented to different groups. For example, for the utterances in (41), Group 1 heard (41a) and (41b) while Group 2 was presented with (41a) and (41c). The filler trials in (42) and (44) were presented to all of the participants.

(41) Target Sentence

$\begin{array}{ccc}\text { a. Jiaozi } & \text { huozhe } & \text { shousi, } \\ \text { dumplings } & \text { or } & \text { sushi } \\ \text { gongfuxiongmao } & \text { dou } & \text { hui } \\ \text { KFP } & \text { DOU } & \text { can } \\ \text { zuo. } & \text { Yejiushishuo } & \\ \text { make } & \text { that is to say } & \end{array}$

"Dumplings or sushi, KFP DOU can make.

That is to say"

b. Gongfuxiongmao hui zuo

$\begin{array}{ccc}\text { KFP } & \text { can } & \text { make } \\ \text { yi } & \text { zhong } & \text { shiwu } \\ \text { one } & \text { kind } & \text { food }\end{array}$

"KFP can make one kind of (the mentioned) food."

$\begin{array}{ccc}\text { c. Gongfuxiongmao } & \text { hui } & \text { zuo } \\ \text { KFP } & \text { can } & \text { make } \\ \text { liang } & \text { zhong } & \text { shiwu } \\ \text { two } & \text { kind } & \text { food }\end{array}$

"KFP can make both kinds of (the mentioned) food."

(42) Filler Sentence

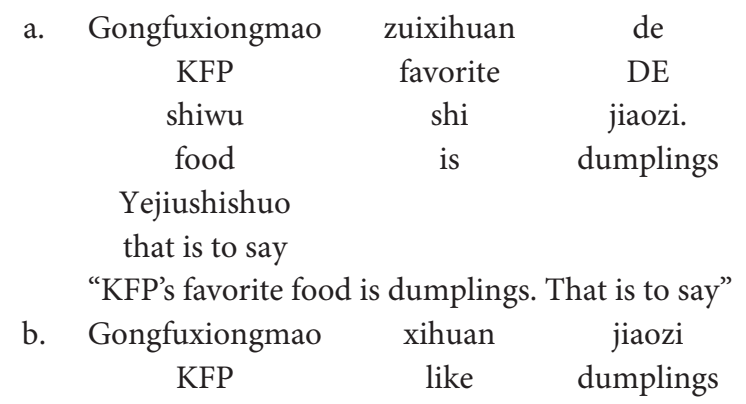

$$
\begin{array}{cc}
\text { chaoguo } & \text { shousi. } \\
\text { more than } & \text { sushi }
\end{array}
$$

"KFP likes dumplings more than sushi."

$\begin{array}{ccc}\text { c. Gongfuxiongmao } & \text { bu } & \text { xihuan } \\ \text { KFP } & \text { not } & \text { like } \\ \text { jiaozi. } & & \\ \text { dumplings } & \\ \text { "KFP doesn't like dumplings." } & \end{array}$

Target Sentence

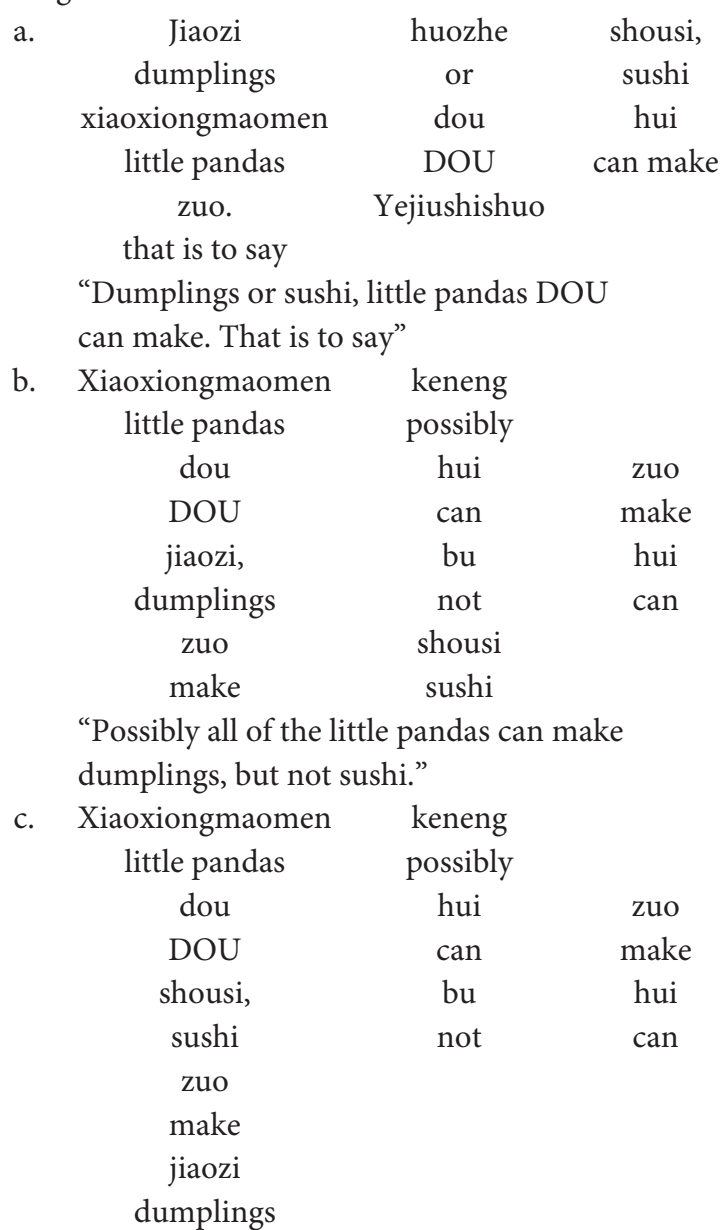

"Possibly all of the little pandas can make sushi, but not dumplings."

(44) Filler Sentence
a. Gongfuxiongmao you liu-ge KFP have six-CL hao pengyou. good friends
Yejiushishuo that is to say
"KFP has six good friends. That is to say"

$\begin{array}{ccc}\text { b. Gongfuxiongmao } & \text { you } & \text { buzhi } \\ \text { KFP } & \text { have } & \text { more than } \\ \text { yige } & \text { hao } & \text { pengyou. } \\ \text { one-CL } & \text { good } & \text { friends }\end{array}$

"KFP has more than one good friends." 


\section{c. gongfuxiongmao mei you KFP not have hao pengyou. good friends \\ "KFP does not have good friends."}

Negative answers (plus correct justifications) to (41b) and positive answers to (41c) were taken as evidence that participants assign conjunctive inferences to the disjunctive phrases in (41a). Positive answers and negative answers to (43b) and (43c) were regarded as evidence that participants assign disjunctive and conjunctive interpretations to the disjunctive phrases in (43a), respectively.

\section{Results}

Table 1 presents the mean and standard deviation of conjunctive readings and disjunctive readings of each group. We analyzed the data with the software package of SPSS 26. Each group's correct answer to the filler trials was above 98\%, which suggested that participants were not challenged by the task. We did not analyze the results of the filler trials in the following.

The conjunctive readings of Group 1 and Group 2 to (41a) and the disjunctive reading assigned to (43a) by Group 3 and Group 4 were analyzed. Data analysis revealed no significant difference between Group 1 and Group 2, $p=0.65$; and no significant difference between Group 3 and Group 4, $p=0.124$. We interpreted our results as evidence in favor of the analysis that when the Q-adverb dou takes scope over disjunctive phrases, they evoke free choice inferences.

\section{DISCUSSION}

Previous studies revealed that, in Mandarin, wh-indefinites and the NPI renhe (English any) are licensed in the same downward entailing linguistic environments (e.g., in the scope of meiyouren "nobody"). In these environments, both wh-indefinites and renhe are typically analyzed as existential/disjunctive expressions. Whindefinites and renhe can also be bound by the Q-adverb dou, which typically takes scope over plural noun phrases to its left. When bound by dou, wh-indefinites and renhe generate universal/conjunctive/free choice truth conditions. This chameleon-like behavior is reminiscent of English any, which is as an existential item/NPI in some linguistic environments, but a universal/free choice item in others. According to one recent approach, NPI any and FCI any are uniformly existential/disjunctive expressions. In its guise as an FCI, any is converted from a disjunction to a conjunction by recursive exhaustification. The same, two-stage process is invoked to

TABLE 1 | Percentage of judgments of participants in Experiment 2.

\begin{tabular}{lccc}
\hline Test sentences & Group & Conjunctive reading & Disjunctive reading \\
\hline Type 1 test sentence & Group 1 & $0.92(0.24)$ & $0.08(0.24)$ \\
& Group 2 & $0.94(0.22)$ & $0.06(0.22)$ \\
Type 2 test sentence & Group 3 & $0.11(0.28)$ & $0.89(0.28)$ \\
& Group 4 & $0.05(0.20)$ & $0.95(0.20)$
\end{tabular}

explain how the universal force of wh-indefinites bound by dou is derived. Specifically, an exhaustivity function ONLY first applies to a set of domain alternatives, yielding suitably enriched alternatives, and then it applies again to the output of its first application. At the second step, the exhaustivity function ONLY negates any of the enriched domain alternatives that are stronger than the original assertion. Adopting this approach leads to an interesting prediction, namely that the Q-adverb dou should be able to bind disjunctive NPs to its left, converting them into conjunctive interpretations.

This approach was further confirmed in the present study using sentences like (45), where the disjunctive phrase jiaozi huozhe shousi "dumplings or sushi" is bound by the Q-adverb dou (in the absence of any plural NP antecedent to the left of dou). The result was taken as evidence that both children and adults generated a conjunctive interpretation of (45), which can be paraphrased as KFP can make dumplings and sushi. In (46), a plural NP xiaoxiongmaomen "little pandas" replaced the singular gongfuxiongmao "KFP" in (45), such that dou had a plural NP to bind in (46), instead of the disjunction phrase. Consequently, the disjunctive phrase was assigned "disjunctive" truth conditions in (46), again by both children and adults. So, sentence (46) was interpreted to mean that the little pandas all can make dumplings, or sushi.

$\begin{array}{cccc}\text { jiaozi } & \text { huozhe } & \text { shousi, } & \text { gongfuxiongmao } \\ \text { dumplings } & \text { or } & \text { sushi } & \text { KFP } \\ \text { dou } & \text { hui } & \text { zuo. } & \\ \text { DOU } & \text { can } & \text { make } & \end{array}$

Literal meaning: "Dumplings or sushi, KFP DOU can make."

Intended: "KFP can make dumplings and sushi."

(46) jiaozi huozhe shousi, xiaoxiongmaomen dumplings or sushi little pandas dou hui zuo.

DOU can make

Literal meaning: "Dumplings or sushi, little pandas DOU can make."

Intended: "The little pandas all can make dumplings, or sushi."

Previous work demonstrated that Mandarin-speaking children of the same age have adult-like command of sentences with wh-indefinites as well as the Q-adverb dou (Zhou and Crain, 2011; Zhou et al., 2013). Taken together, these findings invited us to conclude that, by the age of 4, Mandarin-speaking children have the knowledge of the semantics of dou, including recursive exhaustification. In drawing conclusions from the findings of the present study, however, it is important to be certain that the Mandarin disjunction word huozhe was responsible for the conjunctive interpretation assigned by children and adults.

To appreciate the potential problem, consider the sentences in (47) and (48). We will refer to (47) as a "parallel structure." Despite the absence of the disjunction word huozhe, (47) generates a conjunctive interpretation, just as (48) does. The pertinent observation is that both (47) and (48) contain the 
Q-adverb dou. This raises the possibility that we would have obtained the same findings in our study if we had used sentences without huozhe, such as (47) instead of ones with huozhe, such as (48).

$\begin{array}{ccc}\text { jiaozi, } & \text { shousi, } & \text { gongfuxiongmao } \\ \text { Dumplings, } & \text { sushi, } & \text { KFP } \\ \text { dou } & \text { hui } & \text { zuo. } \\ \text { DOU } & \text { can } & \text { make }\end{array}$

Literal meaning: "Dumplings, sushi, KFP DOU can make."

$\begin{array}{lcc}\begin{array}{c}\text { Intended: "KFP can make dumplings and sushi." } \\ \text { jiaozi }\end{array} & \text { huozhe } & \text { shousi, } \\ \text { dumplings } & \text { or } & \text { sushi } \\ \text { gongfuxiongmao } & \text { DOU } & \text { hui } \\ \text { KFP } & \text { DOU } & \text { can } \\ \text { zuo. } & & \\ \text { make } & & \\ \text { Literal meaning: "Dumplings or sushi, KFP DOU } \\ \text { can make." } \\ \text { Intended: "KFP can make dumplings and sushi." }\end{array}$

It is important to deflect the force of this potential alternative explanation of the findings. We accomplish this by showing that, although the Q-adverb dou is critical for deriving a conjunctive interpretation of sentences with the disjunction word huozhe, it is not critical in deriving the meaning of parallel structure sentences like (47).

To see that dou is a critical ingredient in deriving the conjunctive interpretation of sentences with the disjunction word huozhe, it suffices to compare sentence (48) with sentence (49), which contains huozhe but not the Q-adverb dou (as indicated by the strikethrough). Without dou, the disjunction phrase in sentence (49) takes on "disjunctive" truth conditions, so (49) is true if KFP can only make dumplings, or sushi. Sentence (48) would be false in this circumstance.

$$
\begin{array}{ccc}
\text { jiaozi } & \text { huozhe } & \text { shousi, } \\
\text { dumplings } & \text { or } & \text { sushi } \\
\text { gongfuxiongmao } & \text { hui } & \text { zuo. } \\
\text { KFP } & \text { can } & \text { make }
\end{array}
$$

Literal meaning: "Dumplings or sushi, KFP

can make."

Intended: "KFP can make dumplings or sushi."

It remains to show that the Q-adverb dou is not critical in deriving the conjunctive reading of sentences with a parallel structure. This is accomplished using examples (50) and (51). ${ }^{3}$

$\begin{array}{ccc}\text { Jiaozi, } & \text { shousi, } & \text { gongfuxiongmao } \\ \text { Dumplings, } & \text { sushi, } & \text { KFP } \\ \text { hui } & \text { zuo. } & \\ \text { DOU } & \text { can } & \text { make }\end{array}$

\footnotetext{
${ }^{3}$ Another example is indicted in (i). This is another parallel structure where the $\mathrm{Q}$-adverb dou is not critical in deriving a conjunctive interpretation.

(i) Gongfuxiongmao hui zuo jiaozi, shousi. KFP can make dumplings, sushi
}

\author{
Literal meaning: "Dumplings, sushi, KFP DOU \\ can make." \\ Intended: "KFP can make dumplings and sushi." \\ Jiaozi, shousi, gongfuxiongmao hui \\ Dumplings, sushi KFP can \\ zuo. \\ make \\ Literal meaning: "Dumplings, sushi, KFP can make." \\ Intended: "KFP can make dumplings and sushi."
}

We conclude with a brief comment on how Mandarinspeaking children acquire the knowledge that they displayed in the experiment, i.e., that disjunction phrases yield a conjunctive interpretation in the scope of the universal quantifier dou. First, we want to deal with the possibility that children learn this from the adult input. If they do, then it should be possible to find evidence that adults produce sentences with both the disjunctive word huozhe and the quantifier dou, in sufficient abundance for children to learn the conjunctive interpretation that is assigned by adults in these sentences. To see if this acquisition scenario is on the right track, we conducted a corpus analysis of the 243,593 utterances contained in the six Mandarin Chinese corpora of CHILDES database (MacWhinney, 2000). We found no sentence with both huozhe and dou, although there were 330 sentences containing dou alone, and 11 sentences with huozhe alone. A search of another Chinese corpus TCCM (Taiwan Corpus of Child Mandarin, which includes spontaneous adult-child language samples from nine children, age ranging from 1;6 to 4;3. cf. Cheung et al., 2011) resulted in 2 instances of huozhe and 1143 instances of dou. However, there was no sentence with the combination of huozhe and dou. It should be noted that these are relatively small corpora, and that this could be a sampling error, in which case, examples of huozhe and dou could potentially co-occur in a larger corpus. Nevertheless, this low frequency of relevant input in two separate corpora makes it highly unlikely that children learn the conjunctive interpretation of disjunction based on the adult input. Therefore, we propose an alternative learnability scenario. This scenario is based on the supposition that existential items, such as disjunction words, wh-words, NPIs and FCIs, are innately specified in children's grammar, as part of Universal Grammar. Following Fox (2007), Chierchia (2013), we propose that children initially analyze both $w h$-words and disjunction as existential items, and know that when $w h$-words and disjunction occur in the scope of an exhaustification operator (e.g., the quantifier dou), they yield a conjunctive interpretation using the kind of recursive exhaustification algorithm described in the present study.

\section{CONCLUSION}

A recent proposal by Chierchia (2013) offers a unified analysis of the interpretation assigned to disjunction words, NPIs, FCIs, and the non-interrogative use of wh-phrases in Mandarin Chinese. Evidence in support of the analysis includes the finding that, by age four, Mandarin-speaking children interpret the existential

"KFP can make dumplings and sushi." 
indefinite renhe "any" and wh-words (e.g., shenme "what") as NPIs when they are bound by downward entailing operators, but as FCIs when they are bound by the deontic modal keyi "may" or in combination with the Q-adverb dou "all."

The study extends this line of research by investigating 4-year-old Mandarin-speaking children's interpretation of the disjunction word huozhe "or" in sentences with dou, with and without an intervening plural NP. In sentences with an intervening plural NP, children assigned "disjunctive" truth conditions to disjunction phrases; but when there was a singular intervening NP, children assigned "conjunctive" truth conditions to disjunction phrases. By the age of four, then, children exhibit adult-like knowledge that disjunction phrases generate a conjunctive interpretation in sentences with the Q-adverb dou. This finding is taken as evidence supporting the unified analysis proposed by Chierchia (2013).

\section{DATA AVAILABILITY STATEMENT}

The original contributions presented in the study are included in the article/Supplementary Material, further inquiries can be directed to the corresponding author/s.

\section{ETHICS STATEMENT}

The studies involving human participants were reviewed and approved by the Faculty of Human ScienceHuman Research Ethics Sub-Committee, Macquarie University, Australia. The ethics reference number is 5201200772. Written informed consent to participate in this study was provided by the participants' legal guardian/next of kin.

\section{REFERENCES}

Baker, C. L. (1970). Double negatives. Linguist. Inq. 1, 169-186.

Barker, C. (2010). Free Choice permission as resource-sensitive reasoning. Seman. Pragmat. 3, 1-38.

Carlson, G. (1980). Polarity any is Existential. Linguistic Inq 11, 799-804.

Carlson, G. (1981). "Distribution of free choice any," in Proceedings of Chicago Linguistics Society 17, eds C. Masek, R. Hendrick, and M. Miller (Chicago, IL: Chicago Linguistic Society), 8-23.

Cheung, H., Chang, C., Ko, H., and Tsay, J. (2011). Taiwan Corpus of Child Mandarin (TCCM). Available online at: http://taiccm.org/ (accessed May 01, 2013).

Chierchia, G. (2013). Logic in Grammar: Polarity, Free Choice, and Intervention. Oxford: Oxford University Press.

Crain, S., and Khlentzos, D. (2010). The logic instinct. Mind Lang. 25, 30-65. doi: $10.1111 /$ j.1468-0017.2009.01380.x

Crain, S., and Thornton, R. (1998). Investigations in Universal Grammar: A Guide to Experiments on the Acquisition of Syntax and Semantics. Cambridge, MA: The MIT Press.

Dayal, V. (1995). Licensing any in non-negative/non-modal contexts. Proc. Seman. Ling. Theory 5, 72-93. doi: 10.3765/salt.v5i 0.2694

\section{AUTHOR CONTRIBUTIONS}

SA and PZ conceived the study. SA designed and carried out the experiments, analyzed the data, and drafted the manuscript. SC and $\mathrm{PZ}$ contributed to the writing and revision of the sections "Introduction, Literature Review, Discussion, and Conclusion" part of the manuscript. All authors contributed to manuscript revision, read and approved the submitted version.

\section{FUNDING}

The research was funded by the Postdoctoral Research Foundation of China (2018M643035), Guangdong Planning Office of Philosophy and Social Science (GD19YYY05), and Guangzhou Planning Office of Philosophy and Social Science (2020GZYB49) to SA. These funders provided financial support for the experiments described in the manuscript.

\section{ACKNOWLEDGMENTS}

We sincerely thank all of the participants for their participation in this study. We appreciate the language impairments group of the Guangdong University of Foreign Studies for their assistance with data collection and group members of the language acquisition group at the Macquarie University, specially Dr. Cory Bill, for their feedback, comments and assistance with the research.

\section{SUPPLEMENTARY MATERIAL}

The Supplementary Material for this article can be found online at: https://www.frontiersin.org/articles/10.3389/fpsyg. 2020.609492/full\#supplementary-material

Dayal, V. (1998). Any as inherently modal. Linguist. Philos. 21, 433-476. doi: 10.1023/A:1005494000753

Fox, D. (2007). "Free choice and the theory of scalar implicatures," in Presupposition and implicature in compositional semantics, eds U. Sauerland and P. Stateva (London: Palgrave), 71-120. doi: 10.1057/9780230210752_4

Geurts, B. (2005). Entertaining alternatives: disjunctions as modals. Nat. Lang. Seman. 13, 384-410. doi: 10.1007/s11050-005-2052-4

Horn, L. (1972). On the semantic properties of logical operators in English. Doctoral dissertation. Los Angeles: University of California.

Horn, L. (1989). A Natural History of Negation. Stanford, CA: CSLI Publications.

Huang, A. J., and Crain, S. (2014). Acquisition of the polarity sensitive item renhe 'any' in mandarin chinese. J. Child Lang. 41, 861-889. doi: 10.1017/ S0305000913000275

Huang, H., and Crain, S. (2020). When OR is assigned a conjunctive inference in child. Lang. Acquisit. 27, 74-97. doi: 10.1080/10489223.2019.1659273

Kalish, D., and Montague, R. (1964). Logic: Techniques of Formal Reasoning. San Diego, CA: Harcourt, Brace and World, Inc.

Kamp, H. (1973). Free choice permission, Proceedings of the Aristotelian Society, Vol. 74. Methuen: Aristotelian Society, 57-74.

Klima, E. S. (1964). "Negation in English," in The Structure of Language, eds J. Fodor and J. Katz (Englewood Cliffs: Prentice Hall), 246-323.

Krifka, M. (1995). The semantics and pragmatics of polarity items. Ling. Anal. 25, 209-257. 
Ladusaw, W. (1979). Major Concentration in Syntax and Semantics. Austin, TX: University of Texas at Austin.

Ladusaw, W. (1980). Polarity Sensitivity as Inherent Scope Relations. New York, NY: Garland Publishing.

Lasnik, H. (1972). Analyses of Negation in English. Doctoral dissertation. Cambridge, MA: MIT.

Lee, Y.-S., and Horn, L. (1994). Any as indefinite plus Even. Master thesis. New Haven: University of Yale.

Linebarger, M. (1987). Negative Polarity and Grammatical Representation. Ling. Philos. 10, 325-387. doi: 10.1007/BF00584131

MacWhinney, B. (2000). The CHILDES Project: Tools for Analyzing Talk. New Jersey: Lawrence Erlbaum Associates.

Quine, W. V. O. (1960). Word and Object. Cambridge, MA: MIT Press.

Tieu, L. S. (2010). "On the tri-ambiguous status of any: The view from child language," in Proceedings of the 20th Semantics and Linguistic Theory Conference, eds D. Lutz and N. Li (Washington, DC: CLC Publications), 19-37. doi: $10.3765 /$ salt.v20i0.2568

Vendler, Z. (1967). Linguistics in Philosophy. Ithaca, NY: Cornell University Press.

World Medical Association General Assembly (1964). Declaration of Helsinki: Ethical Principles for Medical Research Involving Human Subjects. Available online at: https://www.wma.net/policies-post/wma-declaration-of-helsinkiethical-principles-for-medical-research-involving-human-subjects/ (accessed July 1, 2018).

Xiang, Y. (2016). "Mandarin particle dou: exhaustification over pre-exhaustified alternatives," in Empirical Issues in Syntax and Semantics 11, ed. C. Piñón (Paris: University Paris), 275-304.
Xiang, Y. (2020). Function alternations of the Mandarin particle dou: distributor, free choice licensor, and 'Even'. J. Seman. 37, 171-217. doi: 10.1093/jos/ ffz018

Zhou, P. (2015). Children's knowledge of wh-quantification in Mandarin Chinese. Appl. Psychol. 36, 411-435. doi: 10.1017/S014271641300 0283

Zhou, P., and Crain, S. (2011). Children's knowledge of the quantifier dou in Mandarin Chinese. J. Psychol. Res. 40, 155-176. doi: 10.1007/s10936-0109161-Z

Zhou, P., Romoli, J., and Crain, S. (2013). “Children's knowledge of free choice inferences," in Proceedings of Semantics and Linguistic Theory 23, ed. T. Snider (Ithaca, NY: Cornell University), 632-651. doi: 10.3765/salt.v23i0. 2657

Zimmermann, T. E. (2000). Free choice disjunction and epistemic possibility. Nat. Lang. Seman. 8, 255-290. doi: 10.1023/A:1011255819284

Conflict of Interest: The authors declare that the research was conducted in the absence of any commercial or financial relationships that could be construed as a potential conflict of interest.

Copyright (c) 2020 An, Zhou and Crain. This is an open-access article distributed under the terms of the Creative Commons Attribution License (CC BY). The use, distribution or reproduction in other forums is permitted, provided the original author(s) and the copyright owner(s) are credited and that the original publication in this journal is cited, in accordance with accepted academic practice. No use, distribution or reproduction is permitted which does not comply with these terms. 